Mens

Revue d'histoire intellectuelle et culturelle

mens

\title{
"Nous sommes l'un des deux peuples fondateurs et nous voulons être traités comme tel " : la référence canadienne-française dans la presse franco-ontarienne (1969-1982)
}

\author{
Serge Miville
}

Volume 18, numéro 1, automne 2017

URI : https://id.erudit.org/iderudit/1062934ar

DOI : https://doi.org/10.7202/1062934ar

Aller au sommaire du numéro

Éditeur(s)

Centre de recherche en civilisation canadienne-française

ISSN

1492-8647 (imprimé)

1927-9299 (numérique)

Découvrir la revue

Citer cet article

Miville, S. (2017). « Nous sommes l'un des deux peuples fondateurs et nous voulons être traités comme tel " : la référence canadienne-française dans la presse franco-ontarienne (1969-1982). Mens, 18(1), 111-148.

https://doi.org/10.7202/1062934ar
Résumé de l'article

Cette étude de la presse franco-ontarienne entre 1969 et 1982 s'intéresse à la persistance de la référence canadienne-française dans les journaux en Ontario français. L'auteur, qui propose une analyse des principaux hebdomadaires francophones de la province et de l'édition du samedi du Droit, tente de faire ressortir les liens de continuité existant entre l'identité franco-ontarienne et l'identité canadienne-française au lendemain des États généraux du Canada français. Le projet de société en Ontario français, loin d'être "normaliste ", s'est largement inspiré d'une certaine interprétation du projet

canadien-français. Les journaux citent, notamment, la thèse des deux peuples fondateurs et le droit d'aînesse des Franco-Ontariens dans la province pour justifier l'attribution de nouveaux droits et de nouvelles institutions à la minorité française en Ontario. 


\section{« Nous sommes l'un des deux peuples fondateurs et nous voulons être traités comme tel ": la référence canadienne-française dans la presse franco-ontarienne (1969-1982) ${ }^{1}$}

Serge Miville

Université Laurentienne

\section{Résumé}

Cette étude de la presse franco-ontarienne entre 1969 et 1982 s'intéresse à la persistance de la référence canadienne-française dans les journaux en Ontario français. L'auteur, qui propose une analyse des principaux hebdomadaires francophones de la province et de l'édition du samedi du Droit, tente de faire ressortir les liens de continuité existant entre l'identité franco-ontarienne et l'identité canadienne-française au lendemain des États généraux du Canada français. Le projet de société en Ontario français, loin d'être " normaliste ", s'est largement inspiré d'une certaine interprétation du projet canadien-français. Les journaux citent, notamment, la thèse des deux peuples fondateurs et le droit d'aînesse des Franco-Ontariens dans la province pour justifier l'attribution de nouveaux droits et de nouvelles institutions à la minorité française en Ontario.

1 L'auteur tient à remercier bien sincèrement les évaluateurs anonymes pour la rigueur avec laquelle ils ont évalué cet article. Nous remercions également Harold Bérubé pour avoir commenté une version antérieure de ce texte. 
L'identité en Ontario français traverse présentement une période de réinterprétation historique. Dans un article récent, Michel Bock cerne deux principales tendances dans les études sur l'identité francoontarienne ${ }^{2}$. Une première, celle de la " rupture ${ }^{3}$ ", constate l'émergence d'une identité francophone qui est autonome du Canada français au lendemain des États généraux du Canada français de 1966, de 1967 et de 1969. Chaque province se serait dotée de sa propre culture autonome, elle-même soutenue par un nouveau réseau institutionnel propre à la minorité provinciale. Pour les "rupturistes ", si on nous permet ce néologisme, la question du Canada français cesse de se poser en raison de la rupture qui intervient entre les minorités et le Québec. L'année 1969 marque ainsi le début d'un renouveau identitaire, d'une rupture profonde. Ces nouvelles identités coupent le cordon ombilical de la référence canadienne-française ${ }^{4}$. D’autres, "révisionnistes 5 ", de leur propre aveu, tendent à normaliser l'expérience franco-ontarienne, à limiter le particularisme du parcours francophone et à l'inscrire dans le récit moderniste occidental. Selon eux, la « référence » canadienne-française trahirait la « nostalgie » des chercheurs qui l'évoquent. L'approche cherche donc à libérer les

2 Michel Bock, «Des braises sous les cendres : l'Ontario français et le projet national canadien-français au lendemain des États généraux (1969-1991) ", dans Jean-François Laniel et Joseph Yvon Thériault (dir.), Retour sur les États généraux du Canada français, Montréal, Les Presses de l'Université de Montréal, 2016, p. 167-231.

3 Les études classiques qui abondent dans ce sens demeurent celles de Marcel Martel, Le deuil d'un pays imaginé : rêves, luttes et déroute du Canada français, Ottawa, Les Presses de l'Université d'Ottawa, 1997; d'Yves Frenette, Brève histoire des Canadiens français, Montréal, Éditions du Boréal, 1998; et de Michel Bock, Comment un peuple oublie son nom, Sudbury, Institut franco-ontarien et Éditions Prise de parole, 2001.

${ }^{4}$ Il s'agit ici de la conscience historique du groupe. Voir Fernand Dumont, Genèse de la société québécoise, Montréal, Éditions du Boréal, 1993.

${ }^{5}$ Le texte le plus évocateur de cette pensée est celui de Linda Cardinal, "Sortir de la nostalgie, en finir avec le ressentiment : les francophones hors Québec et la coopération interprovinciale ", dans Simon Langlois et Jean-Louis Roy (dir.), Briser les solitudes: les francophonies canadiennes et québécoise, Montréal, Éditions Nota bene, 2003, p. 15-31. 
francophones d'un lourd passé qui les empêcherait de s'inscrire dans la modernité plurielle. Une forme de cette « révision » généralement absente des études historiques est également présente dans la littérature socioconstructiviste en Ontario français. Joseph Yvon Thériault et É.-Martin Meunier qualifient ce courant d' "école de pensée " ancrée à Toronto. Selon cette approche, toute norme linguistique et identitaire - d'aucuns diront toute référence historique - brime la liberté de l'individu de se construire une identité autonome et émancipatrice. Ces normes, opprimantes, doivent être déconstruites et évacuées de l'univers discursif afin de libérer l'individu de son carcan normatif. La langue devient alors non plus le véhicule d'une culture collective et d'une histoire commune, mais plutôt une compétence, un moyen de communication additionnel; le français est vu ainsi comme une "valeur ajoutée » chez le citoyen ${ }^{6}$.

Une troisième mouvance épistémologique semble poindre depuis quelques années. Celle-ci cherche à réinterpréter le métarécit identitaire franco-ontarien pour déterrer les éléments de continuité qui ont persisté malgré la « rupture ». On peut mentionner, entre autres, François-Olivier Dorais ${ }^{7}$, qui explore cette facette dans ses études sur l'historien Gaétan Gervais. Gervais, rappelons-le, affirmait que " "l’identité franco-ontarienne", comme "l’identité québécoise", et malgré des ruptures profondes, ne sera jamais que le prolongement de l'“identité canadienne-française", elle-même le prolongement de

${ }^{6}$ Pour une analyse plus complète de cette posture épistémologique, nous suggérons la lecture de Joseph Yvon Thériault et d'É.-Martin Meunier, "Que reste-t-il de l'intention vitale du Canada français? ", dans Anne Gilbert, Linda Cardinal et Joseph Yvon Thériault (dir.), L'espace francophone en milieu minoritaire au Canada : nouveaux enjeux, nouvelles mobilisations, Montréal, Éditions Fides, 2008, p. 205-240.

7 François-Olivier Dorais, Un historien dans la cité : Gaétan Gervais et l'Ontario français, Ottawa, Les Presses de l'Université d'Ottawa, 2016; François-Olivier Dorais, "Gaétan Gervais : témoin et agent d'une mutation référentielle en Ontario français", Mens : revue d'histoire intellectuelle et culturelle, vol. XIII, n 2 (printemps 2013), p. 59-99; François-Olivier Dorais, « Gaétan Gervais : un intellectuel francoontarien entre ruptures et constantes ", dans Laniel et Thériault (dir.), Retour sur les États généraux, p. 109-140. 
l'“identité française " ". Notons que cette « troisième voie » ne postule pas que l'identité canadienne-française se manifeste de manière identique au fil des décennies qui ont suivi la rupture. Elle s'intéresse plutôt aux nombreuses composantes de l'identité franco-ontarienne, qui n’ont pas nécessairement rompu avec l'ensemble des éléments référentiels canadiens-français. Si certains éléments se sont amenuisés au fil des ans - c'est notamment le cas du catholicisme ${ }^{9}$ et de la mission providentielle des Canadiens français -, d'autres demeurent fortement ancrés dans le récit franco-ontarien.

Cet article propose de creuser la persistance de la " référence " canadienne-française dans la presse franco-ontarienne après ce que l'on a nommé la « rupture » du Canada français. La référence, comme l'indique Fernand Dumont, comprend les éléments qui forment la conscience nationale et historique d'un groupe. Ce sont les éléments d'un sentiment d'appartenance qui définissent une communauté et qui permettent d'unir ses membres. Le Canada français traditionaliste se définissait, notamment, par son rapport à l'histoire, son réseau institutionnel et sa mission providentialiste. Cette référence prétend que le pays est composé de deux nations fondatrices : l'une est canadienne-française et l'autre, d'héritage britannique. En raison du droit d'aînesse des Canadiens français, ceux-ci auraient un statut qui les distingue des autres minorités ethniques qui composent le pays. Ainsi, le Canada aurait une obligation envers sa minorité de langue française vivant dans les provinces anglophones. Ce serait illusoire de croire que la référence canadienne-française n'a pas subi de changement en Ontario français avant ou depuis la rupture institutionnelle

\footnotetext{
${ }^{8}$ Gaétan Gervais, Des gens de résolution : le passage du "Canada français" à l' "Ontario français ", Sudbury, Éditions Prise de parole et Institut franco-ontarien, 2003, p. 218.

9 L'évolution du catholicisme franco-ontarien reste à étudier bien que son importance après les années 1960 soit indéniable, notamment, en ce qui a trait au débat entourant la gestion scolaire en Ontario français. Voir Danika Gourgon, "Préférezvous votre ail droit ou votre eil gauche?" : langue et religion dans le débat sur la gestion scolaire en Ontario français (1969-1998), thèse de maîtrise (histoire), Ottawa, Université d'Ottawa, 2016.
} 
des années 1960. Toutefois, notre recherche tend à montrer qu'il existe une filiation avec une certaine idée du Canada français.

Cette recherche s'appuie sur une analyse des principaux journaux de l'Ontario français ${ }^{10}$ de 1969 à 1982. Les éditoriaux, qui représentent l'opinion du journal auprès de ses lecteurs, constituent la source principale de cette étude. L'éditorial permet au journal de communiquer sa vision sur un enjeu particulier. Il s'agit souvent de la vision d'un acteur principal, soit le rédacteur en chef, le propriétaire ou un autre journaliste d'importance. Si les journalistes viennent et partent, les éditorialistes ont tendance à rester et à produire un discours stable dans le temps. Le dépouillement du corpus a été accompagné d'une lecture attentive de l'ensemble des éditoriaux pour la période retenue ainsi que des articles connexes qui portent sur la langue française, les luttes politiques chez les francophones, les réseaux institutionnel et associatif franco-ontarien, le Québec, les minorités linguistiques à l'extérieur de l'Ontario, les gouvernements fédéral, provinciaux et municipaux, les crises politiques (locales et nationales) et les événements culturels. En tout et pour tout, ce sont plusieurs milliers d'articles qui ont été minutieusement scrutés et des centaines qui ont été retenus pour l'analyse.

Les journaux franco-ontariens, qui se multiplient vers la fin des années 1960 et au milieu des années 1970, n’ont jamais réellement abandonné l'ensemble des éléments référentiels canadiens-français bien qu'ils aient contribué à sa reformulation progressive. Pour notre démonstration, nous proposons une analyse qui se décline en trois axes. Nous abordons, premièrement, la relation qui existe entre la

10 Notre étude s'étend aux journaux suivants : Le Nord de Hearst (1976-1982), Le Voyageur de Sudbury (1969-1982) - à l'époque propriété des jésuites -, Le Rempart de Windsor (1969-1982, sauf les années 1974 et 1975, qui n'étaient pas disponibles), propriété jusqu'en 1971 de la Société Saint-Jean-Baptiste de l'Ouest de l'Ontario, jusqu'au moment de son indépendance en 1971 pour obtenir les subventions du gouvernement fédéral, Le Carillon de Hawkesbury (1969-1982), L'Express de Toronto (1976-1982), et l'édition du samedi du Droit d'Ottawa (1969-1982). Ces journaux ont été consultés principalement au Centre de recherche en civilisation canadienne-française ou sur microfilm. 
presse et les politiques fédérales, puis la place du Québec dans les journaux et, enfin, les combats contre le gouvernement provincial afin d'obtenir de nouveaux droits et de nouvelles institutions pour l'Ontario français. Chacun des cas nous permet de mieux comprendre la posture de la presse en relation avec la référence canadiennefrançaise. $\mathrm{Si}$, au départ, les journaux jubilent à l'annonce de la décision du gouvernement fédéral d'instaurer le bilinguisme officiel, ils se ravisent bientôt lorsqu'ils constatent que la politique du multiculturalisme risque de faire perdre au "deuxième peuple fondateur " certains de ses privilèges. Le Québec occupe une place à part dans les pages des journaux. Les positions adoptées par la «Belle Province» y sont scrutées à la loupe. Les journaux sont d'abord hostiles aux politiques linguistiques de la province, mais les crises scolaires ontariennes viennent à bout de leur patience, de sorte qu'ils sont de plus en plus portés à défendre le Québec et son gouvernement péquiste. Enfin, notre section sur les revendications en Ontario montre une presse militante qui estime que les Franco-Ontariens ont des droits que bafoue la Province, notamment au sujet des écoles secondaires. Codifiés ou non, ces droits doivent, selon cette presse, être respectés afin de triompher de l'ancien projet d'assimilation des francophones mené par le Canada anglais.

Notre étude se limite à la période de 1969 à 1982 puisqu’il s’agit, comme l'affirme Bock, d'un nouveau chapitre dans l'évolution de la référence en Ontario français ${ }^{11}$. De son côté, Jean-François Laniel parle d'une "seconde rupture" en raison de l'adoption de la Charte canadienne des droits et libertés ${ }^{12}$. En 1969, lors de l'adoption de la Loi sur les langues officielles, l'élite canadienne-française estime avoir enfin obtenu une reconnaissance historique longtemps convoitée. Le bilinguisme canadien serait ainsi le premier pas vers la reconnaissance

11 Bock, "Des braises sous les cendres ", p. 223.

12 Jean-François Laniel, «De l'Amérique française à la francophonie d'Amérique : la transformation des horizons nationalitaires en francophonie canadienne ", dans Laniel et Thériault (dir.), Retour sur les États généraux, p. 312. Les italiques apparaissent dans le texte originel. 
de la thèse des deux peuples fondateurs. Or, après des années de lutte, l'enchâssement, en 1982, de la Charte canadienne des droits et libertés dans la Constitution, qui codifie noir sur blanc les réels droits des francophonies minoritaires, transforme à jamais les relations possibles entre les membres de la famille canadienne-française et le foyer québécois. En effet, la nouvelle charte confond les droits des minorités de "langues officielles ", que ce soient les anglophones du Québec ou les francophones hors Québec. Les intérêts entre le Québec et les minorités francophones deviennent difficilement conciliables. L'élan politique franco-ontarien ralentit, notamment, au profit de la " judiciarisation ${ }^{13}$ " des luttes et de la « normalisation » de l'identité franco-ontarienne au courant des années $1990^{14}$.

Plusieurs limites s'imposent avant de continuer. Il s'agit ici de sonder le terrain des éditoriaux dans la presse franco-ontarienne. Cette méthode ne permet pas de refléter toute la diversité de la pensée collective en Ontario français. Il demeure néanmoins que ces agents produisent un discours qui se répercute dans l'espace public dont ils font partie. Il s'agit également ici de l'opinion d'une certaine élite composée d'hommes blancs ayant eu accès à une certaine éducation. Aucune voix féminine n’a été repérée dans les sources présentées ci-après. Notre étude ne permet pas, non plus, de mesurer la place occupée par l'Église catholique dans la presse franco-ontarienne, bien que certains membres du clergé participent au débat public. Le père Hector-L. Bertrand, s.j., est d'ailleurs le directeur du Voyageur de Sudbury à partir de 1975. La place de la religion déborde toutefois le cadre de cet article, quoiqu'elle mérite que l'on s'y attarde éventuellement.

${ }_{13}$ Bock fait référence ici à l'étude de Joseph Yvon Thériault, L'identité à l'épreuve de la modernité : écrits politiques sur l'Acadie et les francophonies canadiennes minoritaires, Moncton, Éditions d'Acadie, 1995.

${ }_{14}$ Bock, «Des braises sous les cendres », p. 201-220. 


\section{Une référence qui persiste : le Canada français dans les pages des journaux franco-ontariens}

Le crépuscule des années 1960 est une période mouvementée en Ontario français. L'élite associative franco-ontarienne, ne se laissant pas décourager par le déroulement des États généraux du Canada français ${ }^{15}$, se ressaisit et se retrousse les manches, entre autres, en élargissant le mandat de l'Association canadienne-française d'éducation d'Ontario (ACFEO), qui devient l'Association canadiennefrançaise de l'Ontario (ACFO). Dorénavant, l'organisme parapluie de l'Ontario français est déterminé à investir l'ensemble des sphères d'activité de l'Ontario français, se présentant comme l'intermédiaire principal entre la province et la communauté. L'époque est également connue pour les nouvelles politiques fédérales en faveur des Canadiens français en situation minoritaire, dont, en 1969, la Loi sur les langues officielles et l'engagement du Secrétariat d'État de venir en aide aux minorités à coups de millions de dollars ${ }^{16}$. Les journaux francophones bénéficient largement de ces nouvelles sources de financement. Véritable bouée de sauvetage, l'aide accordée, sous la forme de publicités du gouvernement, permet non seulement la stabilisation de leurs finances, mais également la création de nouvelles publications dans des endroits comme Toronto et Hearst.

\section{L'alliance friable avec le fédéral : entre optimisme et désillusion}

Le gouvernement fédéral est, depuis la fin des années 1960, un des grands alliés des minorités canadiennes-françaises du pays. Opérant une «transformation de l'ordre symbolique du Canada ${ }^{17}$ ", l'État

15 De nombreux membres de l'ACFEO qui ont participé aux États généraux en ont gardé un souvenir amer. Certains témoignages sont présentés dans Marcel Martel et Robert Choquette (dir.), Les États généraux du Canada français, trente ans après : actes du colloque tenu à l'Université d'Ottawa les 5, 6 et 7 novembre 1997, Ottawa, Centre de recherche en civilisation canadienne-française, 1998.

16 Bock, Comment un peuple oublie son nom, p. 51-55.

${ }_{17}$ Marcel Martel et Martin Pâquet, Langue et politique au Canada et au Québec: une synthèse historique, Montréal, Éditions du Boréal, 2010, p. 174. 
central multiplie les interventions auprès d'elles. Les journaux en Ontario français croient alors que la victoire du Canada bilingue et biculturel pointe à l'horizon. L'optimisme règne au moment de l'adoption de la Loi sur les langues officielles. Claude Lavergne, éditorialiste au Voyageur, affirme que «le pays [est] lancé sur la voie pouvant conduire à une unité véritable ${ }^{18} »$. Cependant, les politiques du gouvernement n'obtiennent pas qu'un accueil favorable. Si le bilinguisme est vu d'un bon œil, le multiculturalisme est reçu avec hostilité.

L'une des propositions entourant le bilinguisme officiel porte sur la composition de districts bilingues en ce qui a trait à l'offre de services. Or, ce découpage linguistique pose de sérieux problèmes dans certaines régions francophones. Paul Huneault, nom de plume de Marcel Desjardins ${ }^{19}$, relève dans Le Carillon de Hawkesbury que des endroits comme Saint-Boniface au Manitoba pourraient être privés de services en français, car Winnipeg n’a pas le nombre de francophones requis pour devenir un district bilingue. L'éditorialiste dénonce également le fait que l'ensemble du Québec deviendrait un énorme district bilingue et que peu de régions à l'extérieur de la province obtiendraient la désignation ${ }^{20}$. Huneault y voit une injustice à l'égard du Canada français.

Après quelques années d'application insatisfaisante de la Loi sur les langues officielles, l'optimisme de 1969 fait abruptement place à la désillusion, et les éditorialistes francophones de la province s'impatientent. Marcel Gingras, dans Le Droit d'Ottawa, somme le gouvernement de faire preuve de plus de dynamisme dans sa promotion du bilinguisme. Léditorialiste nous apprend que le Toronto Star a qualifié la politique de gaspillage d'argent, notamment, en raison de

18 Claude Lavergne, "Après la langue, le pain et le beurre », Le Voyageur, 8 janvier 1969, p. 4.

19 Desjardins doit utiliser un nom de plume puisqu’il écrit également dans La Presse. Merci à Jean-Maurice Filion, ancien éditorialiste au Carillon, de nous avoir informé de ce fait.

20 [Marcel Desjardins], «Le rapport Duhamel », Le Carillon, 13 mai 1971, p. 5. 
la formation offerte à des fonctionnaires plus âgés ${ }^{21}$. Desjardins cache mal son découragement concernant l'application timide du bilinguisme : " $[\mathrm{P}]$ ourquoi avoir fait cent ans pour en arriver là? ", se demande-t-il. Visiblement, certains journaux franco-ontariens estiment que le gouvernement fédéral a accouché d'une souris ${ }^{22}$. Les articles pullulent d'ailleurs au sujet de la résistance anglophone au bilinguisme que dénonce, bien entendu, la presse franco-ontarienne ${ }^{23}$. Bien que l'adoption du bilinguisme constitue, dans les médias écrits franco-ontariens, une reconnaissance de la légitimité des Canadiens français à titre de peuple fondateur, il n'en est pas ainsi du côté du Secrétariat d'État du pays. La presse commence à se faire du souci.

Les journaux doivent se préparer à une autre déception. Croyant que le bilinguisme représente la première étape vers la reconnaissance officielle de la dualité nationale, ils font part de leur inquiétude devant la montée du multiculturalisme. Conformément à la référence canadienne-française, les journaux estiment que les Franco-Ontariens font partie des deux peuples fondateurs du pays et qu'ils ne formeraient donc pas une minorité comme les autres. C'est pourquoi Joseph Bourdon, du journal Le Voyageur, se permet une salve en 1969 contre les groupes de pression ukrainiens qui sont hostiles à la reconnaissance du caractère bilingue du pays : «Nous comprenons mal, écrit-il, que la minorité ukrainienne au Canada, ou toute autre minorité d'ailleurs, puisse s'opposer à ce que le français et l'anglais soient des "langues officielles" ». Selon Bourdon, la critique des groupes ukrainiens vou-

${ }^{21}$ Marcel Gingras, "Un thème électoral, le bilinguisme ", Le Droit, 20 octobre 1972, p. 6.

22 [Marcel Desjardins], "À pas de tortue ", Le Carillon, 21 décembre 1972, p. 5.

23 "Une réalité : l'anglicisation du Canada (2) », Le Droit, 9 février 1974 [page introuvable en raison de la qualité du microfilm]; Pierre Tremblay, «Bilinguisme: entre le rêve et la réalité », Le Droit, 23 mars 1974, p. 6 ; "Aux échelons supérieurs de la [f] onction publique : une progression francophone décevante selon J.-R. Gauthier ", Le Droit, 31 mai 1975, p. 3; "Selon un juge de Kitchener : "Le Canada, madame, c'est un pays anglais" ", Le Voyageur, 4 août 1976, p. 8; "SALTZMAN : le Québec français; le Canada anglais ", Le Voyageur, 4 août 1976 , p. 8. 
lant qu'une telle politique marginalise les groupes ethniques du Canada serait " ridicule " et " insensé[e] ». Le bilinguisme s'inscrit dans une logique précise qui « [exige] que l'anglais et le français soient des langues officielles pour la bonne raison que le Canada a été découvert, fondé et développé par des gens parlant l'anglais et le français $^{24}$ ». Bourdon reprend l'argument, cher aux Canadiens français, selon lequel le particularisme des francophones est une caractéristique inaliénable du Canada.

En 1975, une rumeur circule à propos du financement des minorités françaises, qui serait jumelé à l'enveloppe destinée au multiculturalisme et aux groupes ethniques ${ }^{25}$. Rappelons que la politique du multiculturalisme, adoptée en 1971, promeut une certaine vision du pays. Ainsi, selon cette politique, celui-ci serait composé d'une grande mosaïque de cultures et d'une seule nation canadienne, ce qui compromet le statut particulier des Canadiens français ${ }^{26}$. C'est le père Noël Fortier à Sudbury qui mène l'attaque. Dans un éditorial signé le 13 août 1975, le Québécois d’origine somme les Franco-Ontariens de résister à cette idée, car « $\mathrm{M}$. Trudeau et son gouvernement porteront aux yeux des historiens, la responsabilité d'avoir ironiquement porté un coup politique très dur à la grande entreprise du bilinguisme et à son corollaire pourtant normal, le biculturalisme ${ }^{27}$ ». En effet, le gouvernement Trudeau n'a jamais réellement cherché à promouvoir le biculturalisme. Il existe donc une certaine confusion chez Fortier quant à l'intention véritable du premier ministre canadien.

Le multiculturalisme fait peur, puisqu'il porte atteinte au peu de privilèges qu'estiment avoir les Franco-Ontariens. Le Droit affirme,

\footnotetext{
${ }^{24}$ Joseph Bourdon, "Au Canada ou en Ukraine? ", Le Voyageur, 8 janvier 1969, p. 4. Nous soulignons.

25 Noël Fortier, "En marge du [c]olloque d'Elliot Lake : "Un peuple tenace et tanné"... qui a peur de manquer de pain ", Le Voyageur, 22 octobre 1975, p. 4.

${ }^{26}$ Germain Dion, « Le gouvernement continue d'accorder une très haute priorité au bilinguisme ", Le Droit, 11 octobre 1975, p. 4.

27 Noël Fortier, «Dire non au multiculturalisme », Le Voyageur, 13 août 1975, p. 4.
} 
en effet, que la politique risque de provoquer " "l'aplatissement des différences" à l'égard des minorités officielles [par rapport aux autres minorités ethniques] " et l'élimination du particularisme canadienfrançais. Germain Dion, du Droit, craint que son adoption mène à une accélération de l'assimilation vers la majorité anglophone ${ }^{28}$. Pour Dion, la différence entre les minorités ethniques de la mosaïque canadienne et les minorités "officielles ", nommément les FrancoOntariens, est évidente. À Toronto, après avoir accueilli de manière fort positive la politique du multiculturalisme ${ }^{29}$, L'Express fait volteface. Profitant de l'élection québécoise de 1976 et de la montée du Parti québécois dans les sondages, l'hebdomadaire dénonce la " politique d'immigration aberrante " du Canada et le fait que certains néo-Canadiens vont "inviter, nous, les canadiens-français [sic], à retourner "en France". [...] Voilà le multiculturalisme $e^{30}$ ".

La place des francophones minoritaires dans le débat public demeure une source de frustration pour plusieurs chroniqueurs. François Taisne, du Toronto Express, signe un billet cynique affirmant que l'élection d'un gouvernement péquiste était nécessaire pour que le pays manifeste un intérêt quelconque à l'unité nationale :

[...] il est curieux que l'on s'inquiète $[\ldots]$ de l'avenir de l'« [u]nité » canadienne depuis la victoire du Parti [q]uébécois. [...] on [n']en avait pas fait de drames [lors de] l'imposition de législations provinciales restrictives affectant l'avenir national des Canadiens français [avec] le Manitoba Official Language Act, en 1890, le fameux Règlement XVII de l'Ontario [...] et combien d'autres législations... Par contre, chaque geste visant à rétablir un cadre vital minimum pour les Canadiens français suscite une atmosphère de crise nationale. $O$ tempora! $O$ mores $^{31}$ !

28 Dion, "Le gouvernement continue ».

29 [François Taisne], "Tel est pris dans les élucubrations celui qui avait cru prendre ", Le Toronto Express, 19 mai 1976, p. 3. Il s'agit du premier numéro du journal.

${ }^{30}$ François Taisne, "Les élections au Québec ", Le Toronto Express, 3 novembre 1976, p. 3.

31 François Taisne, "Pour une "Révolution [t]ranquille" ", Le Toronto Express, 18 novembre 1977 , p. 4. 
Taisne dénonce ce qu'il considère être le peu d'engagement fédéral auprès des minorités linguistiques avant l'apparition et la popularisation du mouvement souverainiste québécois.

De nombreuses réactions suivent la publication dans les journaux du document Un choix national. Ce document émanant du gouvernement fédéral, qui vante la richesse de la culture et de l'histoire bilingue et multiculturelle de la nation canadienne, n'inspire pas énormément confiance. Si Pierre Tremblay, du Droit, croit qu'il s'agit d'un bon point de départ ${ }^{32}$, Paul Tanguay, du journal Le Nord, est plutôt critique à l'égard d'un gouvernement qui " a ouvert toute grande sa machine à propagande ${ }^{33}$ ". L'éditorialiste affirme qu'il s'agit plutôt de «belles paroles et [de] promesses sans conséquences ", qui amènent les gens à se "fondre dans la belle mosaïque qui, à la fin, parle l'anglais ${ }^{34}$ ». Force est de constater que le multiculturalisme n'arrive pas, au cours des années 1970, à se faire de nombreux adeptes dans la presse franco-ontarienne.

Le ton monte d'un cran lors de la publication du rapport de la commission Pépin-Robarts en 1979, qui propose une décentralisation importante de l'État fédéral. Cette approche signerait l'arrêt de mort des minorités françaises, si l'on en croit la presse et le réseau associatif francophones du pays ${ }^{35}$. Il faut dire que la Commission ne s'était pas faite d'amis à Moncton en 1978 quand le coprésident, Jean-Luc Pépin, a affirmé que les Acadiens "manqu[aient] de colonne vertébrale » pour faire respecter leurs droits linguistiques ${ }^{36}$.

32 Pierre Tremblay, "Un choix national », Le Droit, 22 juin 1977, p. 6, cité dans Le Voyageur, 29 juin 1977, p. 4.

33 Paul Tanguay, "Si peu que point... ", Le Nord (Hearst), 29 juin 1977, p. H2.

34 Ibid.

35 Linda Cardinal et Marie-Ève Hudon, «La dualité linguistique au Canada : les réactions des minorités francophones hors Québec à la Commission de l'unité canadienne, 1977-1979 ", dans Jean-Pierre Wallot (dir.), Le débat qui n’a pas eu lieu : la Commission Pépin-Robarts, quelque vingt ans après, Ottawa, Les Presses de l'Université d'Ottawa, 2002, p. 48-58, coll. "Amérique française ».

36 Pierre Tremblay, "L'échine courbée », cité dans Le Voyageur, 8 février 1978, p. 4. L'éditorialiste en profite pour fustiger Pépin, affirmant que la situation minoritaire est « enracinée si loin dans l'histoire " qu'il faut que les gouvernements offrent activement des services en français. 
Selon l'historien Fernand Harvey, la commission Pépin-Robarts a exposé « le fossé politique qui existait à l'époque entre les intérêts du Québec et des communautés francophones minoritaires ${ }^{37}$ ". La lecture des éditoriaux de Tremblay nous en convainc également. Ce dernier appuie notamment « l'attribution au Québec d'une autorité spéciale en matière culturelle ${ }^{38} »$. Toutefois, la Commission n'a pas proposé d'asymétrie dans la dévolution de pouvoirs. Tremblay doit donc avouer qu'en matière de droits linguistiques pour les minorités, cela dépend de la "bonne volonté des provinces ${ }^{39}$ ", qui, d'ailleurs, ne semble pas déborder du côté de l'Ontario si l'on s'en tient aux crises scolaires qui déferlent partout dans la province.

L'échec du référendum sur la souveraineté-association incite le gouvernement Trudeau à mettre sur pied le processus de rapatriement de la Constitution du Canada. Si Le Carillon, toujours préoccupé par l'unité nationale, se réjouit d'un potentiel rapatriement unilatéral ${ }^{40}$, le jésuite Hector Bertrand, du Voyageur de Sudbury, est plus prudent : "Nous vivons présentement des heures critiques, écrit-il, les heures les plus critiques de notre histoire depuis notre défaite sur les plaines d'Abraham, en septembre $1759^{41}$ ». Il faut donc, selon lui, demeurer vigilants. Le projet de rapatriement inquiète le jésuite, car ses répercussions pourront être aussi tragiques que celles de la Conquête. Le 29 octobre, il renchérit :

Nous voulons, nous les Francophones hors-Québec [sic], être traités d'égal à égal [...]. Nous sommes l'un des deux peuples fondateurs et nous voulons être traités comme tel. À mon avis, si le $[\mathrm{p}]$ remier ministre du Canada pouvait accepter de traduire dans LA constitution cette conception de DEUX PEUPLES

37 Fernand Harvey, «La Commission P[é]pin-Robarts, le Québec et la francophonie canadienne ", dans Wallot, Le débat qui n’a pas eu lieu, p. 41.

38 Pierre Tremblay, "Un nouveau contrat de société" (2) : des propositions innovatrices ", Le Droit, 27 janvier 1979, p. 6.

39 Ibid.

${ }^{40}$ Jean-M. Filion, "Le rapatriement : un bon départ ", Le Carillon, 10 septembre 1980, p. A5.

${ }^{41}$ Hector Bertrand, «Des heures critiques », Le Voyageur, 22 octobre 1980, p. 4. 
FONDATEURS d'égal à égal et, du coup, la nécessité d'un statut particulier pour le Québec, les Francophones du Canada, Québec inclus, appuieraient massivement ses positions ${ }^{42}$.

Bertrand puise entièrement dans l'imaginaire nationaliste du Canada français pour défendre un projet de rapatriement qui serait acceptable pour les minorités françaises. Le Canada doit admettre qu'il est composé de deux peuples fondateurs et reconnaitre que le Québec demeure une société distincte du reste du pays. Lorsqu'il devient clair que le processus de rapatriement n'inclut pas ces éléments jugés essentiels, le jésuite, visiblement frustré, en vient à la conclusion que le premier ministre a trahi les francophones du pays ${ }^{43}$. Au lendemain de l'adoption de la Loi constitutionnelle à Londres, Bertrand a la mine basse :

Les Canadiens français seront-ils vraiment chez eux dans ce Canada [...] ? [...] Pouvons-nous vraiment être chez nous en Ontario? [...] Mais non! Quelles festivités douloureuses! Pouvons-nous vraiment être chez nous au Québec, quand les Québécois, nos ancêtres, ont tout simplement été écrasés, méprisés, trahis même dans le mode de rapatriement de cette Constitution [?] [...] Quelle division non seulement douloureuse et pernicieuse, mais [une] division qui ne sera pas si tôt corrigée. Vraiment, nous aurions espéré davantage d'un homme de la qualité de l'Honorable Pierre Elliot $[\mathrm{t}]$ Trudeau $^{44}$.

La défaite du Québec est donc également celle de l'Ontario français et du Canada français en général, selon Bertrand. L'échec est total, quoique son homologue du Carillon, Jean-Maurice Filion, estime toujours que le premier ministre vise juste. Les garanties juridiques inscrites dans la nouvelle Charte canadienne des droits et libertés viendront renforcer les institutions francophones. " $[\mathrm{A}]$ près tout,

42 Hector Bertrand, "Ce fameux rapatriement ", Le Voyageur, 29 octobre 1980, p. 4. Les majuscules sont de Bertrand.

43 Hector Bertrand, «Trudeau trahit les francophones », Le Voyageur, 21 janvier 1981.

44 Hector Bertrand, «Renouveler le fédéralisme! Dans l'amitié ou la soumission? ", Le Voyageur, 29 avril 1981, p. 4. 
écrit Filion, nous ne sommes pas sous l'épée d'un ayatollah ${ }^{45}$ ! „ Filion est prêt à accepter le " minimum » de droits afin de maintenir l'unité nationale du pays. "Le reste sera pour la prochaine fois ${ }^{46}$ ". En somme, il est irréaliste, d'après l'éditorialiste hawkesbourgeois, de négocier avec les péquistes : "[T] oute entente avec le gouvernement du Québec sera pratiquement impossible tant et aussi longtemps que le Parti québécois détiendra le pouvoir dans cette province ${ }^{47}$. "Cette prise de position radicale demeure marginale dans l'univers médiatique franco-ontarien. Elle montre néanmoins une division en Ontario français quant à l'accueil des décisions prises par l'État central sur les questions constitutionnelle et linguistique.

\section{Le Québec dans les journaux}

Contrairement à ce que laisse entendre la thèse de la rupture du Canada français, le Québec demeure présent dans le paysage médiatique franco-ontarien ${ }^{48}$. La presse ne fait pas exception. La position des journaux concernant le Québec varie selon les années. On peut constater que de nombreux articles sont hostiles aux politiques et aux revendications linguistiques du Québec à la fin des années 1960. Mais la grogne franco-ontarienne se tempère pour se transformer en appui aux demandes québécoises vers le milieu des années 1970, particulièrement en raison d'une succession de crises

${ }^{45}$ Jean-M. Filion, «Et ça recommence! », Le Carillon, 30 septembre 1981, p. A5.

46 Ibid.

47 Jean-M. Filion, "Un pas dans la bonne direction », Le Carillon, 11 novembre 1981, p. A5.

${ }^{48}$ Il est intéressant de souligner que les transformations au Québec sont momentanément vues comme des signes d'émancipation par certains intellectuels franco-américains. Voir Alexandre Patenaude, Le Travailleur et les intellectuels de la survivance franco-américaine face au déclin des communautés francophones de la Nouvelle-Angleterre (1945-1978), mémoire de maîtrise (histoire), Sherbrooke, Université de Sherbrooke, 2015, notamment le chapitre IV, «Entre effervescence et agonie : les intellectuels franco-américains face aux mutations québécoises et américaines », p. 144-198. Patenaude estime toutefois qu'il y a un réel fossé entre les intellectuels qui gravitent autour du Travailleur de Worcester et la population franco-américaine. Leur espoir n’a pas su raviver la " Franco-Américanie ». 
scolaires en Ontario, qui nourrissent le cynisme dans la presse francoontarienne.

Vers 1969, les journaux espèrent toujours obtenir une reconnaissance officielle du Canada français en tant que peuple fondateur. Cet optimisme explique en grande partie les critiques qui sont formulées contre l'aménagement linguistique de la «Belle Province ». D'après le professeur Hugues Albert, de l'Université Laurentienne, « [1]'unilinguisme [français] ", qui semble s'installer au Québec, "n'est pas justifiable dans le contexte nord-américain et encore moins dans celui du Canada ${ }^{49}$ ", car ce serait une preuve de " chauvinisme ", qui aurait pour résultat d' " élargir le fossé qui nous séparent $[s i c]^{50}$ ". À Ottawa, le mouvement McGill français et la croisade d'un conseiller scolaire contre l'établissement d'une école secondaire de langue française à Toronto sont dénoncés comme des "manifestations d'étroitesse d'esprit [...] contre le groupe linguistique minoritaire ${ }^{51}$ ".

Il faut aller dans l'Est ontarien pour rencontrer une voix dissonante en 1969. Marcel Desjardins, qui écrit également dans La Presse, salue la tentative de franciser le Québec et réfute les critiques contre le Québec :

C'est facile, ça fait bien et c'est courant, affirme le journaliste, de blâmer le Québec par les temps qui courent. Et malheureusement, beaucoup trop de francophones vivant hors du Québec éprouvent de la honte à l'endroit de la Belle [P] rovince. Trop nombreux sont ceux qui rougissent lorsque le Québec parvient à se tenir debout tant bien que mal [...] en dépit d'un climat politique agité. On n’a pas à rougir lorsque les Québécois réclament le français prioritaire au Québec; lorsque des Québécois désirent franciser davantage Montréal et le Québec. C'est légitime. Il s'agit d'une question de vie ou de mort $^{52}$.

${ }^{49}$ Hugues Albert, «Unilinguisme ou bilinguisme », Le Voyageur, 26 mars 1969, p. 4.

50 Hugues Albert, "Le projet de loi 63 ", Le Voyageur, 12 novembre 1969, p. 4.

51 "De l'étroitesse d'esprit à Montréal et à Toronto ", Le Voyageur [s. d.], cité du Droit, 14 mai 1969, p. 4.

52 Marcel Desjardins, "Sur un discours de M. Fernand Guindon », Le Carillon, 10 avril 1969, p. 11. 
En 1974, le propriétaire du Carillon est également en faveur des lois linguistiques québécoises. Faire du français la langue officielle de la province serait "normal ", car le Québec ne ferait qu'imiter ce qui a lieu de facto en Ontario ${ }^{53}$. Même Le Voyageur change son fusil d'épaule, alors que Raymond Maynard signe un éditorial qui marque un changement dans la position de l'hebdomadaire concernant les politiques linguistiques du gouvernement québécois :

En Ontario[,] que vous soyez francophone ou non, vous devez apprendre l'anglais. La langue de travail est l'anglais. La langue anglaise est obligatoire dans les écoles françaises alors que le français ne l'est pas dans les écoles anglaises. Qui proteste sur ce sujet? Personne. La province est anglophone [tout comme le Québec est francophone $]^{54}$.

La Charte de la langue française, ou loi 101, est amplement commentée dans les journaux franco-ontariens. En effet, plusieurs appuient l'initiative de normaliser la langue française sur le territoire québécois. L'éditorialiste Pierre Tremblay, du Droit - un chroniqueur québécois, il faut le rappeler - propose un texte, reproduit dans Le Voyageur, dans lequel il affirme que la loi reflète l'esprit de la commission Laurendeau-Dunton " dont on a malheureusement oublié le deuxième volet, celui où les commissaires du temps ont conclu qu'il y avait au Canada deux sociétés différentes ${ }^{55}$ ». L'Express de Toronto y voit le résultat du refus du gouvernement Trudeau de légiférer en faveur des droits collectifs :

Quelle farce [ce Trudeau] ! La question de la langue d'enseignement n’est bien que la micro-facette d'une problématique générale, celle de la plausibilité de l'existence d'une nation canadienne-française minoritaire éparpillée, dominée, fractionnée et, hors du Québec,

53 André Paquette, "Le français la langue officielle au Québec... Pourquoi pas? ", Le Carillon, 7 mars 1974, p. 1.

${ }^{54}$ Raymond Maynard, "Pourquoi tant de "Placottage" [sic] ? ", Le Voyageur, 29 mai 1974, p. 4.

55 Pierre Tremblay, «Le français à venir ", Le Voyageur, 13 avril 1977, p. 4. L'article original parait dans Le Droit, 6 avril 1977. 
épuisée, administrée par des niveaux de gouvernement inaptes à répondre à leurs aspirations ${ }^{56}$.

À Hearst, Jean Gagnon souligne l'importance des institutions de langue française pour la population minoritaire. Dans le contexte franco-ontarien des crises scolaires qui caractérisent les années 1970, il affirme que le Québec, « malgré sa [loi 101], a depuis fort longtemps compris que l'éducation était la clé pour permettre à sa minorité de survivre ». Les anglophones de la province, indique-t-il, ont un meilleur accès à l'enseignement dans leur langue que les FrancoOntariens ${ }^{57}$.

En dépit de l'appui assez généralisé à la législation linguistique, Le Carillon fait bande à part. L'éditorialiste Filion se prononce tardivement, en 1981, au sujet de ces lois, en affirmant qu'il « ne [veut] pas chez nous [de] lois qui vont nous interdire [...] d'afficher dans une seule langue ${ }^{58}$ \%. Il défend ici l'idée selon laquelle l'Ontario, contrairement au Québec, offre l'occasion aux commerces d'afficher en français uniquement, sans ajouter la langue de la majorité. À la suite de ce que l'on a appelé la « nuit des longs couteaux », en 1981, l'éditorialiste renchérit :

Cette [loi 101], ne l'oublions pas, a enlevé certains droits que les citoyens d'ailleurs au Canada arrivant au Québec n'ont plus. Les autres provinces n'ont pas [...] enlev[é] des droits à quiconque récemment. [...] Davis n'a pas de lois qui empêchent les francophones arrivant d'ailleurs au Canada d'inscrire leurs enfants dans des classes françaises ${ }^{59}$.

Filion affiche clairement son hostilité à l'égard des lois linguistiques québécoises, craignant notamment qu’elles entraînent des représailles

56 François Taisne, "Une proposition fédérale inacceptable ", Le Toronto Express, 16 septembre 1977, p. 4.

57 Jean Gagnon, " Paradoxe à Queen's Park ", Le Nord (Hearst), 26 septembre 1979, p. H6.

58 Filion, "Et ça recommence! », p. A5.

59 Filion, "Un pas dans la bonne direction », p. A5. 
contre les minorités canadiennes-françaises. Il demeure que Le Carillon fait cavalier seul dans cette croisade contre le Québec. Les autres journaux estiment que c'est de bonne guerre d'affirmer la prédominance du français dans la province.

Les journaux qui sont en faveur des politiques linguistiques ne vont toutefois pas jusqu’à cautionner la souveraineté du Québec. Le projet indépendantiste se heurte aux aspirations de la minorité francoontarienne, qui estime nécessaire d'avoir un Québec fort pour se protéger de l'assimilation canadienne. René Lévesque devient donc persona non grata pour plusieurs éditorialistes au lendemain de la fondation du Mouvement souveraineté-association. Le chef péquiste est sévèrement critiqué à Sudbury : «[S] uggérerait-il que nous nous laissions assimiler? ", demande-t-on dans un éditorial. L'auteur ironise sur le fait que Lévesque ignore l'histoire franco-ontarienne, « car c'est bien la preuve la plus vivante de l'avenir de la Confédération ${ }^{60} »$. Le groulxiste Fulgence Charpentier abonde dans ce sens. « Les 600,000 Canadiens français de l'Ontario, témoins et héros de tant de luttes, apprécieront peu cette condamnation à mort [de Lévesque], mais ils en ont entendu bien d'autres ${ }^{61}$. "

De son côté, Le Carillon aborde la question de manière plus émotive, indiquant que l'option souverainiste est injuste puisqu'elle impose une " frontière identitaire " qui n'a pas raison d'exister entre Canadiens français. André Paquette se demande " quel est le FrancoOntarien qui n'éprouve pas dans le fond de son cœur un énorme déchirement, une profonde douleur » au sujet de la question nationale au Québec. « [L]e Canada français, ce n'est certainement pas seulement le Québec ", car, pour Paquette, "nous sommes aussi Canadiens français que les René Lévesque ou Michel Chartrand ${ }^{62}$ ". Rupture ou non, Le Carillon cherche à maintenir les cadres du Canada français.

\footnotetext{
60 "Merci, M. Lévesque », Le Voyageur, 29 janvier 1969, p. 4.

${ }^{61}$ Fulgence Charpentier, "Les "placotages" de M. Lévesque ", Le Droit, 18 janvier 1969, p. 6.

${ }^{62}$ André Paquette, «Lettre d'un père à son fils ", Le Carillon, 13 octobre 1971, p. 5.
} 
Il est néanmoins intéressant d'observer la réaction de Paul Tanguay, dans Le Nord de Hearst, à la victoire péquiste de 1976. Celui-ci appuie la décision des Québécois d'élire un gouvernement souverainiste en raison de ce qu'il considère être l'hostilité historique des Canadiens à leur égard. Selon lui, le peuple québécois se fait «depuis toujours [...] manipuler et charrier de tout côté6 ${ }^{63}$ ». Il estime qu'il faut être solidaires des Québécois :

Les francophones du nord de l'Ontario ne peuvent pas rester indifférents [...]. Un peuple au seuil d'accéder à son indépendance ne se limite pas aux frontières politiques. Notre langue, nos coutumes, notre culture sont celles de nos grands-pères qui sont venus de la Gaspésie, de la Beauce ou de l'Outaouais. Un sentiment d'appartenance nous relie étroitement au peuple québécois qui choisira d'ici peu son avenir. [...] Dans les mois qui viennent se dessinera notre destin[,] mais nous ne pouvons ignorer les liens étroits qui nous unissent dorénavant avec le peuple québécois. Et il s'agit de solidifier ces liens avant qu'une frontière politique nous sépare... ${ }^{64}$

Le Québec et l'Ontario français font donc partie de la même trame historique pour Tanguay. Cette filiation doit, selon l'éditorialiste, perdurer au-delà des conséquences politiques d'un éventuel Québec indépendant.

L'offensive souverainiste du Québec au lendemain de l'élection d'un premier gouvernement péquiste secoue Le Carillon et son président, André Paquette. L'hebdomadaire est particulièrement virulent dans ses critiques contre le mouvement indépendantiste de la province et rappelle, quelques mois après l'élection, la politique éditoriale du journal, et ce, à la une :

LE CARILLON est un journal indépendant en politique [qui ...]

[c] roit que la place des Canadiens-français $[s i c]$ est partout au

63 Paul Tanguay, "Des liens étroits nous unissent », Le Nord, 17 novembre 1976, p. $\mathrm{H} 2$.

64 Ibid. 
Canada, d'un océan à l'autre, et nous sommes, par conséquent, opposés à toute idée de séparer notre pays. Nous croyons que notre intérêt réside dans un Canada uni et nous jugeons qu'il est de notre devoir de combattre tout[e] idée de séparatisme ${ }^{65}$.

L'attention de Paquette se tourne vers le premier ministre Bill Davis :

Il faut que l'Ontario et les provinces anglophones du Canada montrent aux Canadiens et surtout aux Québ[é]cois que les francophones sont non seulement bienvenus dans les autres provinces[,] mais qu'on les traite bien. Qu'on fasse qu'ils se sentent non pas seulement " tolérés " mais respectés et [comme] des partenaires égaux de cette confédération, de ce Canada qui nous tient tant à cour ${ }^{66}$.

D'autres croient néanmoins que l'on doit être pragmatique compte tenu de la situation politique du pays. Alors que Le Carillon, fidèle à ses habitudes, monte aux barricades contre la tentative d'instaurer un régime de souveraineté-association, Jean Gagnon, du Nord, est plus nuancé : " [L] es francophones [...] auront avantage à profiter du débat dans le seul but de faire préciser les positions des gouvernements canadiens et ontariens [sic] [à leur égard] ${ }^{67}$ ". Hector Bertrand, le jésuite et rédacteur en chef du Voyageur à Sudbury, pèse également ses propos. Il affirme tout d'abord qu'il « veu[t] demeurer fédéraliste ». Toutefois,

[...] si les adversaires du Parti [q]uébécois ne présente[nt] pas une formule à la fois réaliste et efficace pour permettre aux [f] rancophones et aux [a]nglophones de vivre harmonieusement dans un Canada uni, [il se] verrait forcé, peut-être, de modifier $[s]$ on attitude ${ }^{68}$ !

65 André Paquette, « Notre politique éditoriale », Le Carillon, 23 mars 1977, p. 1.

${ }^{66}$ André Paquette, " Pour sauver la Confédération : l'Ontario doit donner l'exemple ", Le Carillon, 23 mars 1977, p. A5.

${ }^{67}$ Jean Gagnon, "Le référendum et les Franco-[O]ntariens ", Le Nord (Hearst), 14 novembre 1979 , p. H6.

${ }^{68}$ Hector Bertrand, "Le livre du Parti québécois : un document euphorique et ambigu ", Le Voyageur, 16 janvier 1980, p. 4. 
Il ne faut cependant pas surestimer l'importance de la menace d'appuyer l'option souverainiste. Bertrand cherche surtout à faire avancer ce qu'il affirme être ses quatre exigences auprès du Canada pour sauver la Confédération, soit "l'égalité des deux peuples fondateurs ", un pays bilingue "en théorie et en pratique ", le «droit d'être chez moi partout au pays ", ainsi qu' " un Canada [uni], non dans la soumission des nôtres, mais dans la justice, dans le respect et la dignité de ma personne, de ma langue et ma culture ». La solution à la crise de l'unité nationale doit, d'après lui, inclure une nouvelle entente avec le Québec "qui se rapproche singulièrement de l'indépendance - sans toutefois être l'indépendance ${ }^{69}$ ".

Enfin, le Québec demeure très présent dans l'univers de la presse franco-ontarienne. En règle générale, les journaux acceptent que la province légifère en faveur du français, mais ils s'inquiètent de l'option souverainiste. Les médias écrits tiennent néanmoins à un lien culturel fort entre le Québec et le Canada français. Certes, le Québec est un État avec ses propres priorités. La presse estime toutefois nécessaire qu'une relation persiste au-delà des crises politiques qui secouent le pays.

\section{Les luttes sur le front ontarien}

La province reste le principal champ de bataille de l'Ontario français. Après des décennies de luttes, entre autres, contre l'infâme Règlement XVII ${ }^{70}$, l'Ontario français obtient enfin le droit à des écoles secondaires publiques de langue française en 1969. Or, le système scolaire ontarien demeure compliqué. Il existe à l'époque deux types de conseils scolaires. Le premier, public, est financé intégralement par l'État. Le second, séparé celui-ci, réunit l'ensemble des écoles catholiques de la province et obtient un financement

${ }^{69}$ Hector Bertrand, «Le référendum : ma position », Le Voyageur, 7 mai 1980, p. 4. Les caractères gras sont de Bertrand.

70 Michel Bock et François Charbonneau (dir.), Le siècle du Règlement 17, Sudbury, Éditions Prise de parole, 2015, coll. " Agora ». 
partiel du gouvernement. Ce sont également les écoles de choix de nombreux Franco-Ontariens, malgré le coût des études au niveau secondaire. Qui plus est, la gestion scolaire demeure souvent dominée par des conseillers anglophones qui, soucieux du prix de la construction de nouvelles écoles, ne sont pas toujours enclins à établir des écoles de langue française. Cela se traduit par de nombreuses crises scolaires, dont les plus connues sont celles de Sturgeon Falls et de Penetanguishene. Les journaux s'impliquent dans les luttes scolaires et linguistiques de la province et appuient largement les revendications de la communauté minoritaire.

\section{Les conflits et les victoires sur le plan de l'éducation}

La mémoire du projet canadien-français est toujours présente lorsque vient le temps de revendiquer des droits linguistiques dans les journaux franco-ontariens. La communauté de Sturgeon Falls, une ville mono-industrielle située entre Sudbury et North Bay, est l'une des premières victimes des lacunes de la loi 141 de 1969, qui donne aux Franco-Ontariens le droit d'obtenir des écoles de langue française. On peut lire dans les pages du Voyageur, en janvier 1969, un appel à la création de ces institutions pour permettre aux FrancoOntariens « de [s']épanouir selon [leurs] normes, [leurs] habitudes, [leur] culture et [leurs] traditions ${ }^{71} »$. L'école bilingue de la ville ne correspondrait pas à ce profil, puisqu'une minorité d'étudiants, soit 300 , sont anglophones. «Si un seul élève dans la classe est de langue anglaise, [l'enseignement se donne] dans cette langue ${ }^{72}$. " Aux yeux du journaliste, cette situation est anormale et exige un remède. Le droit à l'école s'inscrit ainsi dans la protection de la dualité nationale du pays.

Certains opposants aux écoles de langue française estiment que celles-ci ne seraient pas en mesure d'offrir un enseignement de l'anglais

\footnotetext{
71 «Écoles françaises ou bilingues?", Le Voyageur, 22 janvier 1969, p. 4.

72 Ibid.
} 
adéquat aux élèves ${ }^{73}$. L'hebdomadaire se fait toutefois rassurant : " [L]e bilinguisme, dans ces écoles de langue française est chose certaine. Ce qu'il faut déterminer maintenant, c'est à savoir si on veut que les élèves sachent bien le français ou non ${ }^{74}$ ! » Bref, il faut que l'assimilation soit endiguée.

La population francophone de Sturgeon Falls s'impatiente de l'inaction du conseil scolaire de Nipissing, situé à North Bay. Lorsque les parents somment Queen's Park d'agir, le gouvernement demeure discret, affirmant que le dossier est de compétence régionale plutôt que provinciale ${ }^{75}$. Refusant de plier l'échine, la communauté se mobilise et organise une assemblée publique. Or, le directeur du principal employeur, la compagnie Abitibi Pulp and Paper, Cam Barrington, menace de fermer l'usine si l'entreprise doit verser de nouvelles taxes scolaires pour la construction d'une école ${ }^{76}$. Le Voyageur ne tarde pas à dénoncer l'ingérence de Barrington :

Les francophones de Sturgeon Falls [...] ne sont jamais allés vous dire $[\ldots]$ comment administrer votre barque; ils n'ont jamais tenté de définir pour vous quels étaient vos propres besoins [...] Arrêtez, de grâce, de les provoquer ${ }^{77}$.

L'hebdomadaire reproduit également un texte de Marcel Gingras, du Droit, qui se porte à la défense des parents de Sturgeon Falls. La question serait de "grande urgence » et nécessiterait une intervention gouvernementale immédiate par le ministre de l'Éducation de l'époque et futur premier ministre, Bill Davis ${ }^{78}$. Face au mutisme

73 David Welch montre que cet argument a été utilisé lors de la crise scolaire de Sturgeon Falls. David Welch, "La lutte pour les écoles secondaires francoontariennes : une nouvelle perspective ", Revue du Nouvel-Ontario, ${ }^{\text {os }} 13-14$ (1991), p. 109-131.

74 "Appel aux Canadiens-français ", Le Voyageur, 29 janvier 1969, p. 4.

75 "L'école secondaire française de Sturgeon Falls : appel à Davis ", Le Voyageur, $1^{\text {er }}$ avril 1970 , p. 1.

76 "Assemblée publique à Sturgeon Falls : limitation du droit de parole ", Le Voyageur, 8 avril 1970, p. 1.

77 "Le ridicule, 2 édition ", Le Voyageur, 8 avril 1970, p. 4.

78 Marcel Gingras, "Le français, c'est aussi pour Nipissing ", Le Droit [s. d.], cité dans Le Voyageur, 8 avril 1970. 
de la province, le journaliste Hubert Potvin hausse le ton dans l'édition du 15 avril 1970 du Voyageur en déclarant que l'enjeu est une question d'unité nationale. Comment peut-on reprocher aux Québécois d'être tentés par la souveraineté alors que le gouvernement de l'Ontario refuse de mettre sur pied une école secondaire de langue française à Sturgeon Falls? Pourquoi un tel acharnement contre les Franco-Ontariens ${ }^{79}$ ?

Sommé d'agir, le ministre Davis fait une annonce, que la presse juge insuffisante. Au lieu de construire une école de langue française, Davis propose une division administrative de l'école. L'école bilingue aurait donc une administration double, à titre de solution temporaire. L'idée est dénoncée comme étant un " compromis malhonnête ", et pour manifester leur désaccord, les élèves francophones choisissent d'occuper l'édifice en septembre $1971^{80}$. L'enjeu devient alors provincial. Si les éditorialistes du Carillon et du Droits'en prennent aux adversaires de l'école, celui du Rempart de Windsor s'inspire de cet exemple pour encourager ses lecteurs à lutter pour une école francophone dans la région. En l'absence de solution immédiate, un individu insère de la poudre à dynamite dans trois enveloppes, qu'il envoie au conseil scolaire de Nipissing, à un conseiller scolaire et à l'hebdomadaire The Nugget de North Bay, afin de dénoncer leur opposition à une école de langue française. Le coup est revendiqué par le «F.L.O. Cellule Ontario », une organisation assurément fictive et vraisemblablement créée de toutes pièces par un ou des malfaiteurs amateurs $^{81}$.

L'éditorialiste Marcel Desjardins du Carillon prend la plume pour appuyer les demandes des parents et des élèves de Sturgeon Falls. Il dénonce le style " pas très efficace et injuste " du ministre

${ }^{79}$ Hubert Potvin, "Oui, comment? ", Le Voyageur, 15 avril 1970, p. 4.

${ }^{80}$ Hubert Potvin, "Compromis malhonnête ", Le Voyageur, 22 avril 1970, p. 4; "Le compromis de Sturgeon Falls ", Le Voyageur, 22 avril 1970.

81 Manifestement inspiré du Front de libération du Québec, le «Front de libération de l'Ontario " n'est, somme toute, qu'une organisation fictive. "Menace de violence à Sturgeon Falls », Le Droit, 18 septembre 1971, p. 1. 
Davis " envers les francophones ». Il s'insurge contre le fait que, selon la loi, les francophones "doivent démontrer à la satisfaction des autorités scolaires locales, souvent les plus préjugées [sic] et les plus étroites d'esprits $[s i c][\ldots]$ qu'ils ont vraiment besoin [d'une école $]^{82}$ ". Selon lui, la loi 141, dans sa mouture actuelle, serait un échec.

Le mouvement de solidarité que provoque la crise scolaire de Sturgeon Falls s'étend à d'autres conflits similaires. C'est notamment le cas à Cornwall après le congédiement, en 1973, de deux enseignants francophones qui ont appuyé le mouvement étudiant en faveur de l'octroi d'une école de langue française. Par opportunisme ou par conviction, 1050 élèves de Sturgeon Falls sèchent leurs cours « en guise de manifestation de solidarité avec les 750 étudiants de l'école secondaire St-Laurent » de Cornwall ${ }^{83}$. Desjardins, dans le Carillon, cache à peine sa frustration au sujet des crises scolaires :

Les anglophones du [conseil scolaire] se conduisent comme si les conflits scolaires de l'école Champlain et de Sturgeon Falls n'avaient pas eu lieu, comme si les francophones n'avai[en]t pas accumulé, lors de ces batailles, des droits acquis importants ${ }^{84}$.

Force est de constater que la presse franco-ontarienne persiste à dénoncer ce qu'elle considère comme un problème systémique, c'està-dire la discrimination que la majorité anglophone exerce à l'endroit des Franco-Ontariens.

La région de Windsor, bien qu'isolée du reste du paysage francoontarien, est également l'hôte d'une lutte pour l'obtention d'une école secondaire de langue française. Si les revendications pour une école de langue française s'amorcent au lendemain de l'entrée en vigueur de la loi 141, l'école secondaire L'Essor ne voit le jour qu'en 1979, dix ans plus tard. Pour Le Rempart, l'hebdomadaire régional,

82 [Marcel Desjardins], «Sturgeon Falls : Davis se dérobe », Le Carillon, 16 septembre 1971 , p. 5.

83 "Les étudiants de Sturgeon appuient Cornwall : boycottage à Franco-Cité [et] une délégation sur les lieux ", Le Voyageur, 4 avril 1973, p. 9.

${ }^{84}$ [Marcel Desjardins], «Le "fair play" de Cornwall ", Le Carillon, 5 avril 1973, p. 5. 
l'école française est essentielle à la survie culturelle des Canadiens français et se justifie en raison de la dualité nationale du pays. Citant les cas de Sudbury et d'Ottawa qui ont déjà des écoles de langue française, le journal estime qu'il est temps que les parents militent activement pour leur propre établissement ${ }^{85}$.

La préservation de la langue et de la culture canadiennes-françaises dans la région est le principal cheval de bataille du journal et des partisans de l'école de langue française. En 1973, Gérard Lemieux, président du Comité consultatif de langue française du conseil scolaire régional et bientôt président de la régionale de l'Association canadienne-française de l'Ontario, indique que la demande des parents n'a rien d'extravagant :

Ce que nous demandons n'est ni plus, ni moins que ce dont jouissent les enfants de nos concitoyens de langue anglaise, c'està-dire une école qui puisse correspondre à nos besoins intellectuels et culturels et aux aspirations vitales de notre jeunesse ${ }^{86}$.

L'école n'est donc pas une simple courroie de transmission des connaissances nécessaires pour favoriser l'intégration de l'élève au marché du travail, mais devient également une institution où peut rayonner non pas seulement la langue, mais la culture des FrancoOntariens. Pour Lemieux, cette école se justifie non pas purement en raison de droits légaux, mais du fait de la dualité nationale et du particularisme franco-ontarien :

Nous sommes ici depuis trois siècles, ici, nous avons construits [sic] notre chez[-]nous. C'est d'ici que s'ouvrent les perspectives d'avenir de nos jeunes. C'est ici qu'a pris naissance, il y a trois cent $[s]$ ans, le « règne » de notre culture. C'est ici que nous tenons à prendre les moyens pour conserver le patrimoine que nous voulons léguer à nos enfants ${ }^{87}$.

\footnotetext{
85 "Une école secondaire franco-ontarienne dans notre région ", Le Rempart, février 1969, p. 1; "Ce genre d'école existe déjà ", Le Rempart, février 1969, p. 4.

${ }^{86}$ Gérard Lemieux, "Oui, nous l'aurons notre école secondaire franco-ontarienne ", Le Rempart, 6 mars 1973, p. 4.

${ }^{87}$ Ibid.
} 
Mais la particularité de la culture canadienne-française est négligée par le conseil scolaire majoritairement anglophone, selon Lemieux, qui rédige un deuxième éditorial sur le sujet en juin de la même année. Les membres de ce conseil ne sont pas en mesure d'évaluer les besoins des Franco-Ontariens pour des raisons culturelles : «Ces autorités scolaires ne comprennent ni la langue, ni les coutumes, ni la mentalité et ni les habitudes de vie des citoyens francophones qu'elles doivent servir ${ }^{88}$.»

Pour Lemieux, le Franco-Ontarien se distingue de son compatriote de langue anglaise tant sur le plan culturel, historique que linguistique. Il serait donc injuste que les conseillers anglophones refusent aux francophones des droits qui ont été acquis en partie en raison de leur présence historique sur le territoire ontarien.

En général, la rhétorique franco-ontarienne sur la question scolaire s'articule autour de deux idées : l'une morale, et l'autre, légaliste. La majorité ne doit pas dominer la minorité linguistique. La dualité nationale et le bilinguisme exigent, sur le plan moral, des concessions, soit des écoles de langue française, afin de freiner l'assimilation des jeunes franco-ontariens. Du côté légal, plusieurs éditorialistes sont frustrés de constater que la loi n’est pas respectée au nom de l'autonomie régionale des conseils scolaires. L'établissement de nouvelles écoles génère des coûts additionnels que ne sont pas toujours prêts à assumer les conseils scolaires.

\section{L'Ontario français contre-attaque}

L'inaction du gouvernement pour garantir les droits scolaires des Franco-Ontariens pousse la presse à intervenir davantage dans le dossier. Placide Gaboury, professeur à l'Université de Sudbury, se lance dans une critique acerbe du premier ministre Davis, anciennement le ministre de l'Éducation de la province :

${ }^{88}$ Gérard Lemieux, "Va-t-on nous soutirer le peu de droits qui nous reste? ", Le Rempart, 19 juin 1973, p. 4. 
Monsieur William Davis ne reconnaît des droits linguistiques qu'aux anglophones; aux francophones il ne reconnaît aucun droit linguistique, aucun droit culturel, aucun droit d'être pleinement eux-mêmes. C'est ce qu[i] veut dire ne pas être en faveur d'une province bilingue. [...] un droit, ce n'est pas une faveur, et M. Davis parle de faveurs à accorder aux francophones. C'est donc l'histoire qui se répète. Va-t-on, M. Davis, revenir aux fameux jours du Règlement 17, où à petite dose et poliment, on étranglait la race canadienne[-] française $[\ldots]^{89}$ ?

La peur d'un "ressac » anglophone, omniprésente à Queen's Park, semble justifier l'inaction du gouvernement. Davis et son caucus adoptent plutôt une politique qualifiée de " petits pas " pour s'assurer de ne pas heurter sa base électorale. Or, le premier ministre ontarien estime toujours que sa province doit jouer un rôle de chef de file dans la cause de l'unité nationale. Alain Dexter, dans Le Droit, en profite pour écorcher le chef progressiste-conservateur lors de son passage à Montréal en $1977^{90}$. La nouvelle se rend jusqu'à Hearst où "l'hypocrisie » de Queen's Park est dénoncée par Paul Tanguay :

Le gouvernement ontarien se moque des Franco-[O]ntariens. Il déclare par le truchement des média[s] qu'il offre un tas de services dans les deux langues [...] mais derrière tout ça se cache une attitude dénigrante. Quand on reçoit les communiqués de presse en français deux semaines en retard... quand, pour être jugé en français $[\ldots]$ on doit attendre des semaines et perdre des journées de travail... [...] souvent il devient beaucoup plus facile de vivre dans la langue de la majorité... ${ }^{91}$.

Cette fois-ci, Le Carillon se jette dans la mêlée pour appuyer ses collègues du Nord. Certes, l'Ontario doit assurer l'avenir de la Confédération, mais André Paquette ajoute que, pour ce faire,

89 Placide Gaboury, "La mauvaise mémoire de Monsieur Davis ", Le Voyageur, 22 février 1978, p. 4.

90 Alain Dexter, "Bilinguisme : Davis peut-il se permettre d'offrir l'Ontario en exemple au Québec? " Le Droit, 5 mars 1977, p. 5.

91 Paul Tanguay, «Le bilinguisme à la Davis », Le Nord (Hearst), 9 mars 1977, p. H2. 
le premier ministre doit régler les questions linguistiques dans sa province $^{92}$. Le contexte amène même l'archevêque d'Ottawa, JosephAlphonse Plourde, à intervenir dans le dossier. Dans une lettre ouverte publiée dans Le Carillon et Le Nord, celui-ci demande à la Province de respecter les droits des Canadiens français, soulignant du même coup l'histoire de la présence française au pays :

C'est donc à partir de ce moment-là [la Conquête] que deux peuples entreprirent, malgré les préjugés de race, de langue et de religion qui prévalaient alors, de bâtir ici un grand pays. [...] Si l'histoire conferre des droits aux fondateurs d'un pays; si elle confere au pays lui-même ses caractéristiques propres, il faut conclure que les deux peuples fondateurs ont ici des droits uniques et égaux et que le Canada est, d'abord et avant tout, un pays bilingue et biculturel. Autrement, comment expliquer que la Constitution ait voulu assurer à ces deux peuples des droits particuliers? [...] La question qui se pose maintenant à la majorité anglophone de l'Ontario est la suivante : veut-elle conserver cette richesse et cette force que constituent la langue et la culture française[s] ? Veut-elle conserver son identité historique? Saurait-elle s'élever au-dessus des anciens préjugés de race, de langue et de religion et demander à son gouvernement d'accorder à sa minorité francophone les moyens dont elle a besoin pour éviter l'assimilation qui la menace? Si elle le fait, elle montrera son respect de l'histoire, son amour de la justice et du fair-play et donnera au reste du pays un signe évident de sa maturité. Si elle ne le fait pas, elle donnera raison à ceux qui croient que le seul avenir qu’a la minorité française en cette province où elle a pourtant travaillé, c'est la disparition à plus ou moins brève échéance ${ }^{93}$.

Plourde énumère trois « urgentes requêtes " pour l'Ontario français : 1) un statut légal pour le français ; 2) des écoles et la gestion scolaire

92 André Paquette, "Situation des Franco-Ontariens : le ministre Lalonde a crevé l'abcès ", Le Carillon, 16 mars 1977, p. A5.

93 J.-A. Plourde, "Lettre aux anglophones ", Le Carillon, 15 mars 1978, p. A5. Le texte de Plourde est reproduit comme éditorial dans Le Nord. Les caractères gras sont de Plourde. 
à Ottawa-Carleton; 3) des services en français dans le secteur juridique et le secteur de la santé. L'intervention d'un membre si haut placé dans la hiérarchie catholique n'est pas banale. Elle s'ancre, de surcroît, dans l'univers référentiel du Canada français en utilisant comme argument la thèse des deux peuples fondateurs. Notons, d'ailleurs, que selon l'archevêque, ce pacte n'a pas été conclu lors de la Confédération, mais existe depuis la Conquête.

Le comble se produit à la suite du dépôt, le 16 mai 1978, d'un projet de loi privé du député libéral d'Ottawa-Est, Albert Roy, exigeant de la Province l'offre de services en français. À la surprise générale, le $1^{\text {er }}$ juin, le texte est adopté en deuxième lecture à l'unanimité par la législature. Le bureau du premier ministre, pris de court par la tournure des événements, émet immédiatement un communiqué indiquant que le gouvernement va rejeter le projet de loi en troisième lecture et "ne prendr[a] pas non plus de mesure pour déclarer le français une langue officielle en Ontario ${ }^{94} »$. Davis torpille donc le projet de loi privé, ce qui fait réagir vivement les journaux franco-ontariens. En effet, Pierre Bergeron, dans Le Droit, qualifie le $1^{\text {er }}$ juin 1978 de « jeudi noir ${ }^{95}$ ". À l'exception du Carillon, qui affirme que c'est un coup monté par le Parti québécois pour déstabiliser la province ${ }^{96}$, la presse est unanime à dénoncer le premier ministre. «[L]e premier ministre, indique Tanguay à Hearst, a osé encore une fois répéter, mais plus clairement que jamais, son opposition à la reconnaissance des droits des francophones en Ontario ». Pour ce dernier, à chaque hésitation de Davis, "l'assimilation gagne du terrain ${ }^{97}$ ».

La plus virulente et cinglante critique vient toutefois de la Ville Reine. Sous l'en-tête de L'Express où figurent les mots : « Je me souviens du $1^{\text {er }}$ juin... ", Roger Besner déclare dans une salve dénonçant la

94 Pierre Bergeron et Pierre Tremblay, "L'espoir et l'affront », cité dans Le Toronto Express, 9 juin 1978, p. 1-2.

95 Pierre Bergeron, "Les lendemains du jeudi noir ", Le Droit, 3 juin 1978, p. 6.

${ }_{96}$ Jean-M. Filion, "Examen de conscience ", Le Carillon, 7 juin 1978, p. A5.

${ }_{97}$ Paul Tanguay, «Les clôtureux : les meilleurs alliés de Davis ", Le Nord (Hearst), 7 juin 1978, p. H6. 
situation " colonisée » des Franco-Ontariens, critiquant particulièrement les Franco-Ontariens associés au Parti progressiste-conservateur de l'Ontario :

Que penser des faux-amis [sic] de la «trempe » de René BRUNELLE [le député de Cochrane-Nord], qui tout en connaissant le coup de théâtre que nous réservait le premier [m]inistre à l'issue du vote, s'évertuait à chanter en Chambre les mérites du projet de loi de Monsieur ROY? Un véritable traitre s'il en est un - et ils pullulent au sein du gouvernement DAVIS. Que penser de cette pléthore de Franco-Ontariens, cadres supérieurs et moyens au sein de diverses [d] irections gouvernementales, presque tous détenteurs de carte de membre du Parti $[c]$ onservateur ${ }^{98}$, qui travaillent et conseillent le Cabinet, non pas pour nous, francophones de l'Ontario, mais pour leur propre avancement dans la hiérarchie gouvernementale [...] ? Nous sommes des colonisés, avec l'esprit de colonisés bien ancré en nous [...]. Comment s'attendre à autre chose qu'à des miettes de la part du [g]ouvernement [...]. Somme toute, notre état de colonisés ne fait que renforcer, en Ontario, le parallèle avec nos confrères québécois [...]. Sauf pour une différence majeur $[s i c]$. Le Québécois a compris, et travaille en ce moment à prendre sa situation en main. [...] force nous est de constater que la seule solution possible, [...] [est de] nous ranger du côté de nos frères québécois, et lutter avec eux à l'édification d'un pays qui lui seul saurait respecter ce que nous sommes : des citoyens d'origine, de tradition et de cœur français, qui en ont marre de lutter pour ce qui, d'office, devrait sauter aux yeux : le droit de cité dans un pays où il fait bon vivre, et non plus vivoter ${ }^{99}$.

Les éditorialistes flirtent donc avec l'idée qu'il existe des « collaborateurs ". Yves Tassé, dans Le Voyageur, continue dans la même

98 Il est évidemment question ici d'Omer Deslauriers et des membres du Conseil des affaires franco-ontariennes.

99 Roger Besner, «Ton existence en Ontario, francophone, n'est pas légale, sembleraitil... Monsieur Davis, votre superch[e] rie nous écœure ", L'Express de Toronto, 9 juin 1978. 
veine, accusant Davis d'être "l'un des grands architectes de notre génocide $\mathrm{g}^{100} »$.

Le ton qu'empruntent certains éditorialistes est, dans certains cas, dramatique. On ne peut raisonnablement pas parler de "génocide " des Franco-Ontariens dans la province, quoique la résistance à offrir une législation substantielle sur les services en français soit tangible. Les exagérations sont également au rendez-vous durant les nombreuses crises scolaires des années 1970. Après des années de démarches infructueuses en faveur d'une école de langue française à Windsor, Le Toronto Express dénonce ce qu'il estime être une "crise contre l'humanité » et accuse les « individus qui s'opposent à notre droit d'existence nationale " de participer à un " "GÉNOCIDE" contre l'humanité101 ». Guy Lacombe, dans Le Droit, affirme qu'il faut se retrousser les manches :

Trop de Franco- $[\mathrm{O}]$ ntariens ont appris à se contenter de peu : à force de manger des miettes, ils ont fini par oublier qu'il y a un gâteau quelque part... Ce sont les gens qui disent qu'il " ne faut quant $[s i c]$ même pas exagérer ", "qu'après tout, on vit en Ontario ", "qu'on est chanceux d'avoir ce qu'on a ", etc. C'est l'attitude suicidaire que souhaitait naguère trouver Lord Durham chez des «Canadiens» du Bas-Canada ${ }^{102}$.

Plusieurs Franco-Ontariens auraient intériorisé une vision misérabiliste et un comportement passif devant le gouvernement ontarien en raison des nombreux échecs pour obtenir des écoles de langue française. Ce rappel de l'histoire, par l'évocation du spectre de lord Durham, vise donc à donner un électrochoc aux militants. Il s'agit également d'une rare présence d'un discours " postcolonial » francoontarien dans les médias, une position qui fera long feu, mais qui

100 Yves Tassé « À monsieur Davis, l'un des grands architectes de notre génocide ", Le Voyageur, 14 juin 1978, p. 4.

101 "La [c]rise de Windsor, crise contre l'humanité ", Le Toronto Express, 9 mars 1977, p. 4. Les majuscules sont de l'auteur.

102 Guy Lacombe, «Il ne faut pas lâcher! », Le Droit, 16 juillet 1977, p. 6. L'éditorial est repris dans Le Nord (Hearst), 20 juillet 1977, p. H2. 
témoigne d'un appétit de donner un sens nouveau aux défis de l'Ontario français.

La crise scolaire de Penetanguishene de 1979-1982 est décrite de façon similaire. La presse y voit un combat entre les "gentils", les « résistants » et les « méchants assimilateurs " du gouvernement. Pour Jean Gagnon à Hearst, l'Ontario poursuit sa « vendetta qui a débuté par l'adoption du [R] èglement $17^{103}$ ". L'inaction de la ministre de l'Éducation, Bette Stephenson, provoque l'ire d'Alain Dexter, du Droit, qui l'accuse d'être " animée par un sentiment anti-francophone" et de condamner les Franco-Ontariens à "l'acculturation ${ }^{104}$ ». Dans le contexte de la crise qui se poursuit et d'un référendum québécois à l'horizon, l'éditorialiste ottavien va même jusqu'à déclarer : « Et s'il s'agissait bel et bien d'une conspiration? Si l[a] ministre de l'Éducation Bette Stephenson était une sorte de Himmler, chargé de l'“extermination" de ces [francophones ...] ${ }^{105}$ ? » La comparaison à l'Allemagne nazie demeure l'hyperbole la plus déplacée à avoir été utilisée dans la presse à cette époque. Elle montre néanmoins combien la frustration est palpable du côté des éditorialistes francophones de la province.

Ce qu'il faut retenir de ces prises de parole sur l'éducation en Ontario français, c'est qu'il se tisse entre les régions de l'Ontario français une certaine solidarité interrégionale. Les revendications auprès de la Province se fondent sur plusieurs références à l'histoire et sur un principe de "droits ", même si ces derniers n'existent pas nécessairement sur le plan juridique. Le " droit » aux services en français ou au bilinguisme dans la province est basé sur la conviction que les Franco-Ontariens forment un peuple égal à la majorité, bien qu'aucune disposition légale ne soit en place pour appuyer cette thèse.

103 Jean Gagnon, «L'école de la résistance », Le Nord (Hearst), 12 septembre 1979, p. H6.

104 Alain Dexter, "L'histoire jugera », Le Droit, 6 octobre 1979, p. 6.

105 Alain Dexter, "Queen's Park n’a rien compris ", cité dans Le Rempart, 5 mars 1980, p. 2. 


\section{Conclusion}

L'étude de la presse franco-ontarienne nous permet de nuancer l'image d'un Ontario français en rupture avec le Québec et avec sa référence canadienne-française pour la période retenue. Pour la presse, les référents historiques canadiens-français permettent de justifier et de légitimer les revendications franco-ontariennes et de souligner le caractère particulier et différent des francophones par rapport aux autres minorités du pays.

Plusieurs éléments de la référence canadienne-française persistent dans l'espace public franco-ontarien. Selon notre analyse de la presse franco-ontarienne, la dualité nationale est au cœur des revendications pour l'obtention d'institutions scolaires ou de droits linguistiques. En effet, la revendication des "droits ", qui sont souvent inexistants dans les lois provinciales et fédérales, fait appel à la notion de « droits historiques " que posséderaient les Franco-Ontariens en tant que peuple fondateur. Ces droits existeraient en raison d'un droit d'aînesse qui remonte à la Conquête et même à Samuel de Champlain. Sinon, c'est en vertu du pacte de 1867 que les Franco-Ontariens peuvent revendiquer des institutions indépendantes de celles de la majorité. Le rapatriement de la Constitution canadienne, toutefois, sape la force de cet argument en codifiant explicitement les droits des «minorités de langue officielle».

En ce sens, le rapatriement de la Constitution et l'enchâssement de la Charte canadienne des droits et libertés changent la donne de manière dramatique pour les minorités françaises du pays. Il devient alors très difficile de lier le sort des Franco-Ontariens aux aspirations nationales des Québécois, car ces deux groupes n’appartiennent plus à la même catégorie juridique. Les droits des francophones hors Québec, en raison de l'article 23 de la Charte, sont intimement liés à ceux des Anglo-Québécois. De fait, sur le plan constitutionnel, les francophones en milieu minoritaire ne sont plus liés à leurs compatriotes québécois. Les effets ne se font pas attendre. Comme le montre Bock, les minorités francophones doivent composer avec des gouver- 
nements québécois successifs qui sont beaucoup moins attentifs à leurs besoins ${ }^{106}$. Le Québec cesse alors d'être un allié des minorités francophones, notamment lorsque vient le temps de défendre les droits linguistiques devant les tribunaux. Soucieux de maintenir son autonomie dans ses champs de compétences, le Québec va, par exemple, s'allier à l'Alberta contre la minorité franco-albertaine durant l'affaire Mahé sur la gestion scolaire. Les victoires juridiques des francophones portent désormais atteinte à l'autonomie québécoise. Qui plus est, les nombreux accords constitutionnels (Meech et Charlottetown) marquent un tournant important dans les relations entre le Québec et les minorités francophones. Ces dernières, souligne Bock, se permettent de virulentes critiques contre leur ancien allié en raison de son indifférence à leur cause ${ }^{107}$. La communauté de destin qui liait l'ensemble des francophones du pays est désormais remise en question.

Les années 1990 constituent ainsi une période d'incertitude identitaire pour l'Ontario français. Dans ses travaux, Bock révèle que l'ACFO, qui représentait jusqu'alors un château fort de la référence canadienne-française, intègre progressivement le discours fédéral sur le multiculturalisme dans sa définition des Franco-Ontariens. L'ACFO élimine, en 1998, l'adjectif « canadienne-française » de son nom dans le but de souligner le caractère pluriel de la francophonie ontarienne ${ }^{108}$. En effet, tout ce qui était vu comme " ethnique " devient, au cours des années 1990 et 2000, synonyme d'exclusion et d'enfermement. Laniel montre aussi, dans son étude sur la Fédération des communautés francophones et acadienne du Canada, que celle-ci intègre progressivement les concepts de francophonie et de francophonie

106 Michel Bock, "La Fédération des francophones hors Québec devant le gouvernement québécois (1976-1991) : groupe de pression ou compagnon d'armes? ", dans Jérôme Boivin et Stéphane Savard (dir.), De la représentation à la manifestation: groupes de pression et enjeux politiques au Québec, XIX ${ }^{e}$ et XX ${ }^{e}$ siècles, Sillery, Éditions du Septentrion, 2014, p. 234-274.

107 Bock, "Des braises sous les cendres ", p. 210.

108 Ibid., p. 218. 
d'Amérique en rejetant les notions de Canada français et de francophonie hors Québec. Ce nouveau paradigme, apolitique et dénationalisé, permettrait de souligner son ouverture à l'Autre et sa participation à l'universel ${ }^{109}$.

Il n'y a pas eu de " grand moment » dans le divorce entre l'Ontario français et son passé canadien-français. Les États généraux du Canada français ont peut-être donné lieu à un affrontement spectaculaire entre les minorités francophones et le Québec, mais la rupture avec la référence canadienne-française n’a pu être consommée lors du rassemblement montréalais. Son récit était nécessaire dans un Canada où les droits culturels et linguistiques des francophones demeuraient ambigus. Sa nature imprécise permettait aux élites de tisser un discours sur les droits des francophones, qui s’ancrait dans une référence indépendante de celle que construisait à la même époque le Canada anglais. En ce sens, la notion des deux peuples fondateurs est demeurée fondamentale chez les éditorialistes franco-ontariens pour justifier le bien-fondé des revendications de la minorité franco-ontarienne pendant la période étudiée. Elle forme la pierre angulaire de l'ensemble des revendications linguistiques à une époque où aucune charte ne protégeait les minorités et où la complétude institutionnelle se gagnait à coups de luttes politiques. L'identité franco-ontarienne, en gestation au cours des années 1970 et 1980, puise à la même source que celle du Canada français et contient donc d'importants éléments de continuité.

109 Laniel, «De l'Amérique française à la Francophonie d'Amérique », p. 297-343. 